\title{
A Study on the Prevention and Control of Major Chronic Non-communicable Diseases Abroad
}

\author{
Zhang Zhuqin, Liu Depei \\ Institute of Basic Medical Sciences, Chinese Academy of Medical Sciences, Beijing 100005, China
}

\begin{abstract}
Major chronic non-communicable diseases, including cardiovascular and cerebrovascular diseases, cancer, respiratory diseases, and diabetes, are prevalent in China and around the world, and pose significant health threats. In order to effectively prevent and control chronic diseases, enhance national health, and promote economic and social development, the Chinese Academy of Engineering launched a project in 2014: International Comparative Study on the Prevention and Control of Major Chronic NonCommunicable Diseases. This project conducted a series of studies on the prevention and control of chronic diseases at home and abroad. Based on the project's report, this paper describes the prevention and control methods and compares the prevention and control systems of various chronic diseases of different countries. This paper then proposes measures and recommendations with the goal of strengthening the prevention and control of chronic diseases in China.
\end{abstract}

Keywords: chronic non-communicable diseases; cardiovascular and cerebrovascular diseases; cancer; respiratory diseases; diabetes; disease control-and-prevention system

\section{General situation of global chronic diseases}

Cardiovascular and cerebrovascular diseases, cancer, respiratory diseases, and diabetes are four major chronic diseases that threaten global health. In 2012, 17.5 million deaths were due to cardiovascular and cerebrovascular diseases [1], and the number is expected to reach 25 million by 2020 [2]. In 2012, there were more than 14 million cases of cancer worldwide. The types of cancer, in descending order of their incidence rates, are lung cancer, breast cancer, colorectal cancer, prostate cancer, stomach cancer, liver cancer, cervical cancer, esophageal cancer, and bladder cancer [3]. Respiratory diseases include chronic obstructive pulmonary disease (COPD), asthma, and lung cancer. Eighty percent of respiratory diseases occur in low- and middle-income countries [4]. In 2013, $8.3 \%$ of adults worldwide (i.e., 382 million people) suffered from diabetes [5].

\section{Global prevention and control of chronic diseases}

\author{
2.1 Prevention and treatment of cardiovascular and \\ cerebrovascular diseases
}

The earliest scientific knowledge about cardiovascular and cerebrovascular diseases came from the Framingham Heart Study. In the 1940 s, the number of deaths from cardiovascular and cerebrovascular diseases accounted for half of the total number of deaths in the United States (US). Due to the lack of understanding about the causes of the diseases, there were no effective treatments. It was in this context that the large-scale Framingham cohort study was initiated. Until now, three large studies have been conducted on this cohort. The results of these studies showed that smoking, low-density lipoprotein (LDL) cholester-

Received date: January 9, 2017; Revised date: March 14, 2017

Corresponding author: Liu Depei, Institute of Basic Medical Sciences, Chinese Academy of Medical Sciences, Professor; Chinese Academy of Engineering, Academician. Major research fields include pathogenesis of cardiovascular disease and gene expression regulation. E-mail: liudp@pumc.edu.cn Funding program: CAE Advisory Project "International Comparison of Prevention and Control Systems of Major Non-communicable Diseases" (2014-XY-44) Chinese version: Zhang Zhuqin et al. A Study on the Prevention and Control of Major Chronic Non-communicable Diseases Abroad. Strategic Study of CAE, https:// doi.org/10.15302/J-SSCAE-2017.02.019 
ol, hypertension, obesity, menopause, and other factors increase the risk of cardiovascular and cerebrovascular diseases, whereas exercise and high-density lipoprotein (HDL) cholesterol are beneficial factors for preventing cardiovascular and cerebrovascular diseases [6]. The concept of "risk factor" was proposed for the first time by the Framingham researchers. Because cholesterol is an important factor in cardiovascular and cerebrovascular diseases, the US established the National Cholesterol Education Program and developed the Adult Treatment Panel (ATP) Guidelines to manage the control of cholesterol levels in adults. ATP I, published in 1988, was the first framework document. ATP II, published in 1993, was supplemented by new experimental evidence for the diagnosis and treatment of cholesterol levels. ATP III, which was published in 2002, integrated the results of a number of statin clinical trials. The US National Cholesterol Education Program has become a worldwide example for cholesterol education and the ATP Guidelines have become the criteria for cholesterol control.

The development of cardiovascular and cerebrovascular diseases is gradual, but the occurrence of a cardiovascular or cerebrovascular event, such as a myocardial infarction or a stroke, is sudden. Therefore, the construction of an emergency rescue system is of great significance. There is a large difference between China and abroad. For example, the success rate of emergency treatment for cardiac arrest is less than $1 \%$ in China, whereas it is $10 \%-30 \%$ in developed countries [7]. With respect to rescue rate and response time, an ambulance arrives at the scene in about 10 minutes in China, on average, whereas it arrives in 7-8 minutes abroad [7]. Awareness of emergency services is low in China, whereas it is relatively high in developed countries. As for resuscitation, only $1.6 \%$ of people in China can correctly perform resuscitation [8]. As for defibrillation outside of the hospital, few people are able to use defibrillators properly, and there are legal issues about using them. In developed countries, including the US and Germany, defibrillators are used more often, and there are legal exemptions that protect rescuers from liability [9]. The treatment of thrombosis also is very important because the immediate treatment of thrombosis influences later treatment effects. It is best to perform thrombolytic therapy within 40-45 minutes. Compared to China, developed countries have better thrombolytic outcomes [10]. Taken together, China's emergency rescue system has a lower efficiency and can be improved substantially. China should further promote the development of its emergency rescue system. In addition, due to high cardiovascular and cerebrovascular events among elderly people, it is necessary to strengthen the care and monitoring of this population.

\subsection{Prevention and treatment of cancer}

According to CONCORD (a Global Cancer Survival Study), the five-year cancer survival rate of lung cancer is $10 \%-20 \%$ worldwide, and it is $30 \%$ in Japan, $24 \%$ in Israel, and $18 \%$ in
China. The five-year survival rate of gastric cancer is $58 \%$ in South Korea, $54 \%$ in Japan, and $31 \%$ in China. The five-year survival rate of adult leukemia is $50 \%-60 \%$ in North America, West Asia, Europe, Oceania and other parts of the world, and is $21.2 \%$ in China [11]. Many methods can be used to treat cancer, including surgery, chemotherapy, radiotherapy, and other treatments, but not all methods are equally efficient. Early screening, diagnosis, and treatment for cancer are very important for cancer prevention and control. The screening methods include: blood tests for a variety of cancers, a B-scan for abdominal tumors, $\mathrm{X}$-ray or low-dosage CT to screen for lung cancer, an anorectal examination for colorectal cancer, a pap smear for cervical carcinoma, and mammography for breast cancer. Internationally, early screening and detection of cancer have achieved good results; therefore, we should encourage early check-ups and screening for cancer. Financial support is recommended to promote early screening for cancer.

\subsection{Prevention and control of respiratory diseases}

The main respiratory diseases are COPD, asthma, and lung cancer. The primary risk factors for respiratory diseases are environmental pollution and smoking. Air pollution can cause an acute exacerbation of COPD symptoms. In developing countries, including China, lack of awareness and lack of treatment of respiratory diseases are important factors contributing to disease epidemics. For example, by the time COPD is diagnosed, it has often reached the later stages of the disease and is difficult to reverse. Hence, early diagnosis and treatment are strongly recommended.

\subsection{Prevention and control of diabetes}

Diabetes is associated with lifestyle factors, including obesity, lack of physical activity, and improper diet. The relationship between diabetes and obesity varies across the world: obesity is associated with diabetes in $30 \%$ of cases of diabetes in China and Japan, $60 \%-80 \%$ of cases in people of European and African heritage, and $100 \%$ of cases in Pima Indians and Pacific Islanders [12]. Improving lifestyle habits, controlling obesity, and increasing exercise are effective measures to prevent and alleviate diabetes. Diabetic complications are important causes of death. Thus, patients with diabetes need to control their blood glucose levels properly, detect diabetic complications, and standardize treatment in order to reduce the hazards of diabetes.

\section{Comparison of the disease control systems of different countries}

\subsection{US prevention and control system: research-oriented, emphasizing health education}

The US disease prevention and control system is based on sci- 
entific research and evidence-based medical practice. It emphasizes health education and health promotion. The US has always been at the forefront of medical research, such as the well-known Framingham study of cardiovascular and cerebrovascular diseases [6]. That study helped health professionals recognize the risk factors for chronic diseases, including cardiovascular and cerebrovascular diseases, and develop guidelines to control the risk factors, such as blood lipids and blood pressure, and maintain exercise. Under the guidelines, government departments, research institutions, clinical hospitals, communities, and schools have attached great importance to health education. The guidelines provide accurate information about disease control for patients and the public. Health education has achieved positive results in that the mortality rate of chronic diseases has been significantly reduced.

\subsection{British prevention and control system: universal health care, layered system}

The United Kingdom has a universal health-care system that is mainly overseen and operated by the National Health Service (NHS). Citizens who have an income are required to participate in social insurance. The NHS has two levels: the first level consists of community medical professionals and family doctors. Patients have to go to this level of hospital first. The second level consists of central hospitals and superior hospitals, which are responsible for emergency and surgical treatment. The NHS system emphasizes health for all people and stresses primary health care, which is crucial for the early diagnosis and prevention of chronic diseases. The NHS is considered to be one of the greatest achievements of the twentieth century in Britain [13].

\subsection{Swedish prevention and control system: a "cradle-to- grave" protection system}

Sweden is a high-welfare state with a "cradle-to-grave" social security system, which is a leader in the prevention and control of disease. In 2009, the total cost of health care in Sweden accounted for $9 \%$ of GDP, of which $80 \%$ was public health costs. There are three levels of government in Sweden (national, county, and municipal) that play roles in disease prevention and control. The primary responsibility for providing health services to the population is at the county level, while health services for the elderly and disabled are concentrated at the municipal level [14]. The measures taken in Sweden have achieved good health outcomes. Sweden ranks high in the world in population life expectancy, and it has high survival rates for patients with coronary heart disease and cancer.

\subsection{Cuba's prevention and control system: focusing on prevention, emphasizing grass-roots implementation}

Cuba is a developing country, but its health care has reached the level of developed countries. The average life expectancy of men and women has reached 78 years and 80 years, respectively. Cuba has a medical care system that is free to all people, and its prevention and control system focuses on disease prevention. In communities, there are family doctors who often do home visits and are concerned about and know the health status of the people who live there. Patients with minor illnesses are treated by family doctors in a timely manner to prevent disease progression to later, serious stages. The risk factors for chronic diseases are well controlled, leading to effective prevention and control of chronic diseases [15].

\subsection{Prevention and control in underdeveloped countries: weak basis, needing more effective control}

Compared with the countries mentioned above, underdeveloped countries, especially poor countries, such as those in subSaharan Africa, are still at a relatively early stage in their prevention and control of chronic diseases. Although the incidence of chronic diseases in these poor countries is not high, chronic diseases have become a heavy burden due to the large population base, the widespread epidemic of tobacco and alcohol, and relative improvements in living and economic conditions in recent years. The disease prevention and control systems are not perfect in these countries due to lack of funds. It is urgent to increase investments, strengthen government leadership, and ask the World Health Organization (WHO) and other international organizations to collaborate with local governments. It is necessary to promote media health education and community health education to raise people's awareness of diseases. In addition, cultural norms are important factors. Smoking and drinking are not only cognitive problems, but are also influenced by cultural and social environments. The government should advocate less smoking and drinking through policies and culturally based promotional efforts.

\subsection{Comparison of preventive and control systems among different countries}

We found that different countries have different medical systems for chronic diseases. However, there are common characteristics among those countries that have achieved good results: i.e., government leadership, focus on primary health care, and emphasis on health education and health promotion.

\section{Suggestions for chronic disease control in China}

Based on the above comparisons and comprehensive analysis, the following measures are put forward to prevent and control chronic diseases. It is suggested that these measures be put into practice in combination with the current reform of the healthcare system. 


\subsection{Promoting primary health care, and boosting the monitoring and management of chronic diseases}

The key elements of chronic disease prevention and control should be included in the baseline level of health care. It should focus on "prevention" and promote insurance, including "medical insurance" and "health insurance." Inpatients are often at the advanced and late stages of their diseases. Hence, it is necessary for healthcare providers to focus on the early stages of diseases and to provide more care for outpatients. Thus, it is a good idea to increase the proportion of outpatient medical reimbursements. As hypertension, diabetes, and hyperlipidemia are important risk factors for chronic diseases, it is essential to increase reimbursements for drugs that treat these risk factors.

\subsection{Increasing the construction of local hospitals, and training and use of general practitioners}

Primary hospitals and local hospitals play important roles in local medical care. To prevent and control chronic diseases, it is important to construct more grass-roots hospitals and community hospitals. It is also necessary to train and make good use of general practitioners, who should be encouraged to monitor and record individuals' health/disease status, including diabetes, hypertension, and hyperlipidemia. To motivate these practitioners, it might be useful to reward them according to the number of people they oversee and the amount of disease information they monitor.

\subsection{Promoting public education and facilitating physical examinations}

Because chronic diseases are lifestyle diseases, it is crucial to strengthen health education and to enhance public awareness of the idea that "chronic diseases are preventable." People should be encouraged to adjust their daily lifestyle behaviors. Middleaged and older populations are susceptible to chronic diseases; therefore, legislation and policies should encourage these people to have physical examinations. In addition, non-profit organizations should be established to facilitate people having physical examinations at a low cost.

\subsection{Promoting early tumor screening}

Early detection and control of tumors are vital; thus, we should promote early screening, diagnosis, and treatment of tumors. Several low-cost methods are effective, such as Bultrasonic scans for abdominal tumors, X-ray screening for lung cancer, and digital examinations for colorectal cancer. Financial support from local governments is recommended to encourage early tumor screening.

\subsection{Building a green life channel}

The incidence of cardiovascular disease events, such as myocardial infarction and stroke, is critical. The time to rescue patients who suffer these events is very short. Therefore, we should improve China's efficiency of emergency rescue. Legislation for emergency rescue is required. Securing and promoting outpatient emergency care is needed. In addition, it is important to monitor and care for older patients at home who are susceptible to cardiovascular or cerebrovascular events.

\section{References}

[1] World Health Organization. Global status report on noncommunicable diseases [EB/OL]. (2014-12-30) [2016-10-12]. http://www. who.int/nmh/publications/ncd-status-report-2014/en/.

[2] Chockalingam A, Balaguer-Vintro I, Achutti A, et al. The World Heart Federation's white book: Impending global pandemic of cardiovascular diseases: Challenges and opportunities for the prevention and control of cardiovascular diseases in developing countries and economies in transition [J]. Canadian Journal of Cardiology, 2000, 16(2): 227-229.

[3] World Health Organization. World cancer report [EB/OL]. (201412-30) [2016-10-13]. http://apps.who.int/bookorders/anglais/detart $1 . j \operatorname{sp}$ ? codlan $=1 \& \operatorname{codcol}=80 \& \operatorname{codcch}=275$.

[4] World Health Organization. Global surveillance, prevention and control of chronic respiratory diseases: A comprehensive approach (2008-09-17) [2016-10-16]. http://www.who.int/gard/publications/ GARD_Manual/en/.

[5] International Diabetes Federation. International Diabetes Federation diabetes atlas [EB/OL]. (2015-11-20) [2016-10-16]. http:// www.idf.org/diabetesatlas/.

[6] Mahmood S S, Levy D, Vasan R S, et al. The Framingham heart study and the epidemiology of cardiovascular disease: A historical perspective [J]. Lancet, 2014, 383(9921): 999-1008.

[7] Huang Z T, Yang Z F. Early warning and integrative control of cardiac sudden death [J]. Practical Journal of Clinical Medicine, 2012, 9(1): 11-15. Chinese.

[8] Wang Y, Li X H, Liu Y Z, et al. Investigation on the public's cognition of cardiopulmonary resuscitation [J]. Journal of Nursing Science, 2007, 22(21): 69-70. Chinese.

[9] Zhang L M. Responsibility exempts of emergency assistance [J] Jianghai Academic Journal, 2014 (6): 133-142. Chinese.

[10] Wang C J, Wang Y L, Xu A D, et al. Establishment of continuous improvement system for thrombolytic medical care of China's acute ischemic stroke [J]. Chinese Journal of Stroke, 2012, 7(8): 636-641. Chinese.

[11] Allemani C, Weir H K, Carreira H. Global surveillance of cancer survival 1995-2009: Analysis of individual data for 25676887 patients from 279 population-based registries in 67 countries (CONCORD-2) [J]. Lancet, 2015, 385(9972): 977-1010.

[12] Gardner D G, Shoback D M. Greenspan's basic \& clinical endocrinology (9th ed.) [M]. New York: McGraw-Hill Medical, 2011.

[13] Guan Z Y, Jiang M H, Liang Z M, et al. The impact on China of 
community health service and general medicine in foreign countries [J]. Guide of China Medicine, 2012, 10(36): 690-692. Chinese.

[14] Xue W J, Wang F, Li C C. Experiences and implications from the cooperation of health care in Sweden [J]. Chinese Primary Health
Care, 2014, 28(5): 11-14. Chinese.

[15] Wang N, Wang J. The history of Cuban health care system and lessons for China $[\mathrm{J}]$. Chinese Journal of Social Medicine, 2009, 26(1): 19-22. Chinese. 